\title{
High-resolution imaging of photoreceptors in healthy human eyes using an adaptive optics retinal camera
}

\author{
P. Tumahai ${ }^{1} \cdot$ C. Moureaux ${ }^{1} \cdot$ M. Meillat ${ }^{1} \cdot$ G. Debellemanière ${ }^{1} \cdot$ M. Flores ${ }^{1} \cdot$ B. Delbosc $^{1} \cdot$ M. Saleh ${ }^{1}$
}

Received: 9 May 2017 / Revised: 21 January 2018 / Accepted: 19 February 2018 / Published online: 11 July 2018

(c) The Royal College of Ophthalmologists 2018

\begin{abstract}
Purpose To determine the effects of age on perifoveal cone density in healthy subjects using adaptive optics. Methods Healthy subjects of various ages were imaged using an adaptive optics retinal camera (RTX-1 ${ }^{\circledR}$ Imagine Eyes, Orsay, France). All patients underwent a comprehensive ophthalmologic examination and retinal imaging using spectraldomain optical coherence tomography (Spectralis ${ }^{\circledR}$, Heidelberg Engineering, Heidelberg, Germany). Cone density together with cone spacing and cone mosaic packing were measured in the nasal and temporal area $450 \mu \mathrm{m}$ from the fovea. A multivariate analysis was performed to determine which of the following parameters were related to a decrease in cone density: age, axial length, central macular thickness, and retrofoveal choroidal thickness.

Results One hundred and sixty-seven eyes of 101 subjects aged 6-78 years were studied. Perifoveal cone density significantly decreased with age $\left(R^{2}=0.17, p<0.01\right)$. Inversely, cone spacing increased with age $\left(R^{2}=0.18, p<0.01\right)$. There was no change in the cone packing mosaic ( $p>0.05$ ). The mean coefficient of variation between fellow eyes was 3.9\%. Age and axial length were related to a cone density decrease, while choroidal and retinal thicknesses did not affect cone metrics in healthy subjects.

Conclusions A moderate perifoveal cone loss occurs with age. The precise consequences of these findings on visual function should be investigated. In addition to a better understanding of normal retinal anatomy, these results could act as a comparative database for further studies on normal and diseased retinas.
\end{abstract}

\section{Introduction}

Neocortical neurons undergo various structural and physiologic changes with aging, resulting in an approximately $10 \%$ decrease in their number across the age spectrum [1].

The question of whether loss of retinal neurons is a characteristic of aging in the human retina remains controversial. The impact of aging on photoreceptor loss in the human retina has been investigated in the past mainly in histological studies on donor eyes, implying a limited number of samples $[2,3]$.

Electronic supplementary material The online version of this article (https://doi.org/10.1038/s41433-018-0140-1) contains supplementary material, which is available to authorized users.

P. Tumahai

tumahaiperle@gmail.com

1 Ophthalmology Department, University Hospital of Besançon, Besançon, Franche-Comté, France
Improvement of imaging device capabilities has enhanced our understanding of the aging effects on the retinal structures through in vivo studies. For instance, the effects of age on retinal nerve fiber layer (RNFL) thickness was documented using both time and spectral-domain optical coherence tomography (SD-OCT). Although the effects were small $\left(R^{2}<0.17\right)$, the thinning of RNFLs corresponded to a loss of retinal ganglion cell axons occurring with age [4]. OCT studies also showed a decrease in the overall macular thickness [5] and thinning in the choroid in normal eyes [6] with age. However, these imaging devices lacked the necessary resolution to image photoreceptors.

By correcting the optical aberrations of the human eye, adaptive optics (AO) allows real-time imaging of the retina with a lateral resolution of approximately $2 \mu \mathrm{m}$, making it possible to image photoreceptors at the cellular level. This imaging modality has already been used to assess cone distribution at the edges of the foveal peak, in normal eyes, using both the AO scanning laser ophthalmoscope (SLO) [7] and the flood illumination AO camera [8-10]. 
Table 1a Characteristics of the different age groups (mean \pm SD $(95 \% \mathrm{CI}))$

\begin{tabular}{lllll}
\hline & $\begin{array}{l}\text { Group } 1<20 \\
\text { years }\end{array}$ & $\begin{array}{l}\text { Group 2 21-35 } \\
\text { years }\end{array}$ & $\begin{array}{l}\text { Group 3 36-50 } \\
\text { years }\end{array}$ & $\begin{array}{l}\text { Group 4 }>50 \\
\text { years }\end{array}$ \\
\hline$N$ & 16 & 44 & 20 & 21 \\
Sex (female/male) & $13 / 3$ & $24 / 20$ & $17 / 3$ & $10 / 11$ \\
OD/OS & $12 / 1$ & $38 / 6$ & $19 / 1$ & $9 / 12$ \\
Age (years) & $13.1 \pm 4.0$ & $26.6 \pm 3.8$ & $42.4 \pm 4.1$ & $60.6 \pm 8.2$ \\
& $(10.9-15.3)$ & $(25.5-27.8)$ & $(40.4-44.4)$ & $(56.9-64.3)$ \\
Axial length (mm) & $23.1 \pm 1.1$ & $23.8 \pm 0.9$ & $23.6 \pm 1.0$ & $23.5 \pm 1.1$ \\
& $(22.4-23.8)$ & $(23.5-24.2)$ & $(23.1-24.2)$ & $(22.8-24.3)$ \\
\hline
\end{tabular}

Table 1b Demographic data and clinical characteristics

\begin{tabular}{|c|c|c|c|}
\hline & Min & Max & Mean \pm SD $(95 \% \mathrm{CI})$ \\
\hline Age (years) & 6 & 78 & $34.7 \pm 16.7(31.4-38.0)$ \\
\hline Axial length(mm) & 21.32 & 26 & $23.6 \pm 1.0(23.3-23.9)$ \\
\hline Density (cells/mm²) & 14054 & 34,780 & $\begin{array}{l}26,166 \pm 3835 \\
(25,409-26,924)\end{array}$ \\
\hline Spacing $(\mu \mathrm{m})$ & 5.95 & 9.4 & $6.9 \pm 0.6(6.8-7.1)$ \\
\hline $\begin{array}{l}\text { Nearest neighbor } \\
\text { analysis (\%) }\end{array}$ & 34.2 & 54.1 & $44.5 \pm 3.7(43.7-45.2)$ \\
\hline $\begin{array}{l}\text { Foveal retina } \\
\text { thickness }(\mu \mathrm{m})\end{array}$ & 201 & 296 & $233 \pm 19(230-236)$ \\
\hline $\begin{array}{l}\text { Subfoveal choroid } \\
\text { thickness }(\mu \mathrm{m})\end{array}$ & 109 & 752 & $346 \pm 97(331-361)$ \\
\hline
\end{tabular}

Several factors have been reported to be related to cone loss including axial length (AL) [11], intraocular pressure [12], retinal diseases [13-17], macular surgery [18, 19], and drug-related causes $[2,20]$. Recent AO studies have suggested that the aging process could also be associated with foveal cone loss [21, 22].

In the present study, we measured cone density in healthy patients and correlated the results with several factors including age and AL, as well as central retina and retrofoveal choroid thicknesses.

\section{Methods}

A power calculation was performed in order to detect a $10 \%$ decrease in photoreceptors with a type 1 error of 5\% and a type 2 error of $10 \%$. It was based on previous data with a mean density in healthy young adults of $25,940 \pm 2872$ $(n=31)$. The results showed that the minimum number of eyes for adequate study power was 73 .

\section{Participants}

One hundred and one healthy subjects from 6 to 78 years of age were studied (mean \pm SD: $34.7 \pm 16$ years): 78 males and 64 females. They all received a complete eye examination, including best-corrected visual acuity (BCVA) measurement and slit-lamp fundus examination. All subjects had BCVA of 20/20 or better. Exclusion criteria for this study included any systemic disease, retinal disease, or spherical equivalent (SE) refractive errors exceeding -12 to $+6 \mathrm{D}$, which is the spherical ametropia compensation range of the $\mathrm{AO}$ device.

Subjects were classified into four age groups summarized in Table 1a.

A single eye from each patient (67 right eyes and 34 left eyes) was selected for studying the modifications with age. Both eyes of 66 patients (132 eyes) were studied to assess intrasubject variation.

To convert density measurements to metric coordinates (in millimeters) from angular coordinates (in degrees), ALs were measured using a biometer (IOLMaster ${ }^{\circ}$ 500, Carl Zeiss Meditec, Dublin, CA, USA). SE refractive errors were measured using the TONOREF II autorefractometer (Nidek, Aichi, Japan) (Table 1b)

Foveal retina thickness (FRT) and subfoveal choroidal thickness (SCT) were visualized using SD-OCT (Spectralis , Heidelberg, Germany) with the enhanced depth imaging system enabled. The protocol consisted of a horizontal $30^{\circ}$ (length) line passing through the fovea with 1024 A-scan/Bscan. At least $50 \mathrm{~B}$-scans per image were averaged by the Tracking ART AM $^{\mathrm{TM}}$ (Automatic Real-Time) software on the SPECTRALIS ${ }^{\oplus}$. SCT was measured manually, in a blind manner, by two graders using the manufacturer's software (Heidelberg Eye Explorer version 1.6.1.0; Heidelberg Engineering, Heidelberg, Germany), with the segmentation lines moved manually to fit the outer border with the RPE/ Bruch membrane complex and the border with the sclera (Figure). In case of a significant difference in the measurements $(>10 \mu \mathrm{m})$, the results were discussed until reaching a consensus. The FRT was measured by moving the vertical marker on the thickness profile module of the Eye Explorer to the AO imaging zone (Supplemental Fig. 1).

Consent forms were obtained from the patients or their legal representative after a full explanation of the procedures and consequences of this study. The study protocol was approved by the local ethics committee and complied with the requirements of the Declaration of Helsinki. 


\section{Adaptive optics imaging}

Eyes were imaged using a flood illumination AO camera (RTX-1 Imagine Eyes TM, Orsay, France). This commercial clinical prototype approved for research use only in Europe is based on a noncoherent flood-illuminated design with an 850-nm central illumination wavelength and a focusing range of $600 \mu \mathrm{m}$, allowing high-resolution imaging of macular photoreceptors. Its low-noise CCD camera has a pixel resolution of $1.6 \mu \mathrm{m}$ and a frame rate of $9.5 \mathrm{fps}$.

\section{Image acquisition procedure}

AO imaging sessions were conducted without any pupil dilation. A three-axis chinrest and a forehead pad system at a 50-mm working distance aligned the subject's pupil with the imaging system's pupil. The subject was asked to stare at an inner central target. The spherical ametropia was adjusted after entering the subject's $\mathrm{SE}$ refractive error. The retina area imaged was centered on the fovea, resulting in a $4^{\circ} \times 4^{\circ}$ square. During AO imaging, the live, wide-field image of the eye allowed direct visualization of the retinal region being imaged. The imaging depth was chosen within the range of $0-80 \mu \mathrm{m}$ in which the live retina images appeared to be the sharpest. On the device control panel, a numerical value informed the operator of the AO correction level. The lower the value was, the better the correction, providing better acquisition quality. During the approximately 30-s acquisition, 40 live high-resolution images of the retina were automatically averaged by the device.

\section{Image processing}

Each series of 40 images acquired by the $\mathrm{AO}$ camera was processed using software programs provided by the system's manufacturer (CK v0.1 and AO detect v0.1, Imagine Eyes, France). These images were registered using a crosscorrelation method and averaged to produce a final image with an improved signal-to-noise ratio. The raw images that showed artifacts due to eye blinking and saccades were automatically eliminated before averaging. For display and printing purposes, the background of the resulting image was subtracted using a Gaussian filter and the histogram was stretched over a 16-bit range of gray levels.

The positions of photoreceptors were computed by automatically detecting the central coordinates of small circular spots whose brightness was higher than the surrounding background level. First, the averaged image, as obtained before background removal and histogram stretching, was further processed using adaptive and multiple-scale digital filters. Then, the local maxima of the resulting filtered image were detected and their pixel coordinates were recorded. The spatial distribution of these point coordinates was finally analyzed in terms of inter-cell spacing, local cell density, and the number of nearest neighbors using Delaunay triangulation and Voronoi diagrams. The area of analysis corresponded to two $90-\mu \mathrm{m}$ squares $\left(0.3^{\circ} \times 0.3^{\circ}\right)$ placed at a fixed distance from the fovea $\left(1^{\circ}\right)$, nasally and temporally along the horizontal axis of the eye studied (Supplemental Fig. 2). The measurement protocol has been previously described [23]. In brief, taking into account the inability of the camera to discriminate individual cones in the foveola, the perifoveal peak density was determined by moving the square horizontally towards the periphery following a $10-\mu \mathrm{m}$ step until reaching the highest density. Cone density (cells/mm [24]), spacing between cones $(\mu \mathrm{m})$, and percentage of cones with six neighbors calculated on Voronoi diagrams (\%) were measured (Fig. 1). To minimize any potential cone undersampling or oversampling made by the automated software, two investigators (CM, MS) counted manually the cones. Any additional round or oval structure of more than $3 \mu \mathrm{m}$ in width with a reflectivity $>50$ ( $0=$ black; $256=$ white $)$ and missed by the automated detection was considered as a cone (Fig. 2). The data presented for the automated and the manual counting correspond to the mean of the nasal and temporal measurements.

\section{Statistical analysis}

All values represent the mean \pm standard deviation. Descriptive statistics and the matched-pairs $t$ test were used for statistical comparisons between fellow eyes, with the two-tailed $p$ value $<0.05$ considered significant. One-way analysis of variance (ANOVA) with Tukey's multiple comparison post-test were used for comparisons between age groups after verifying that the groups had passed the normality test. The parametric correlation with calculation of the Pearson's $r$ was performed. Statistics were calculated using the commercially available software program GraphPad InStat (GraphPad Instat, Inc., San Diego, CA, USA). Linear regression graphs were obtained using GraphPrism 6. A multivariate stepwise linear regression was performed using IBM SPSS statistics 19.0 to determine which factors (Age, AL, RT, CT) had a statistically significant effect (defined as $p \leq 0.05$ ) on cone density. The significant level at entry was 0.15 and for staying in the model 0.05 .

\section{Results}

\section{Age-related changes}

The perifoveal cone density significantly decreased with age. The results were concordant between automated and manual counting $\left(R^{2}=0.16, \quad p<0.01 \quad\right.$ and $R^{2}=0.17, \quad p<0.01$, 


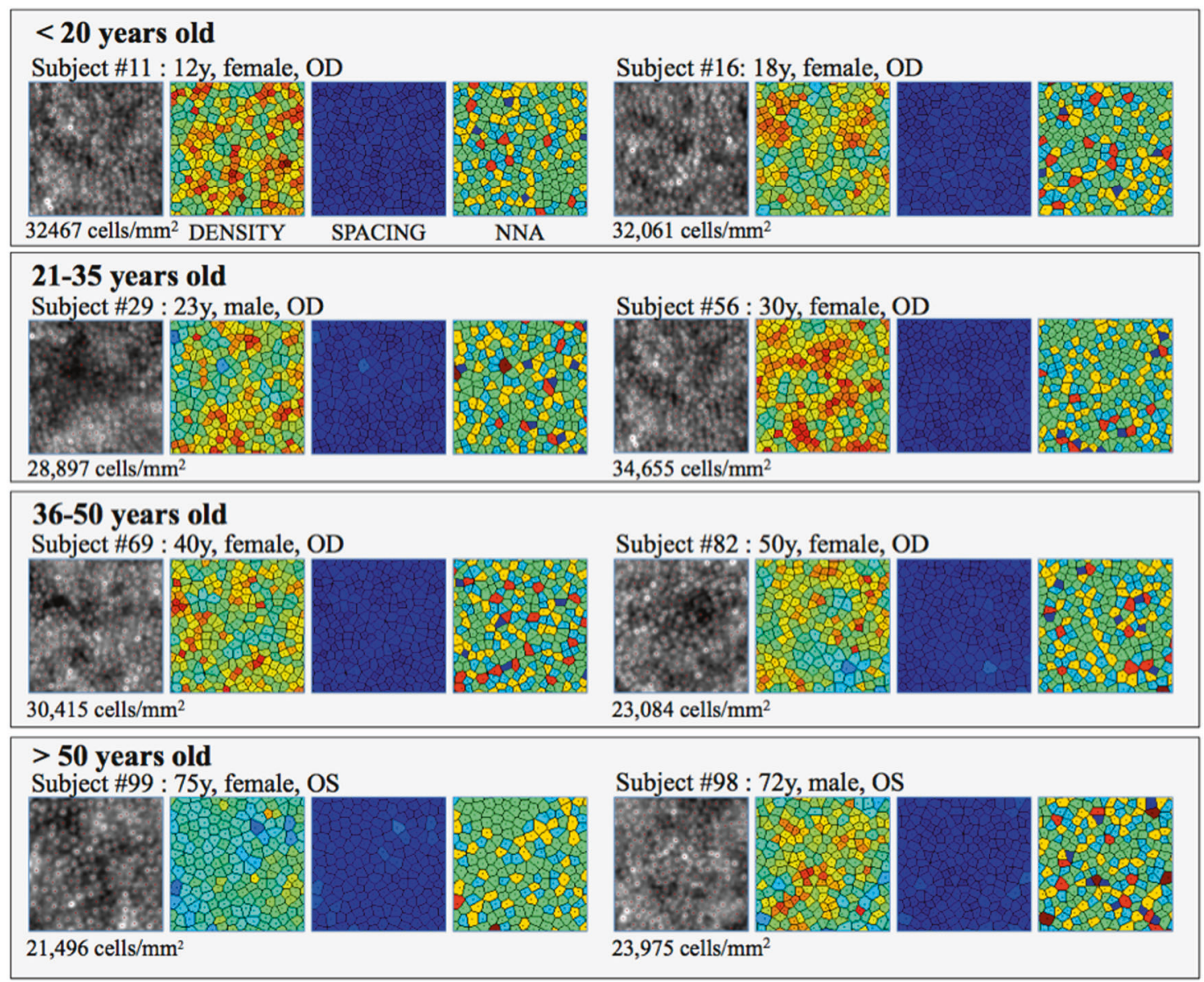

Fig. 1 AO age group samples. NNA nearest neighbor analysis

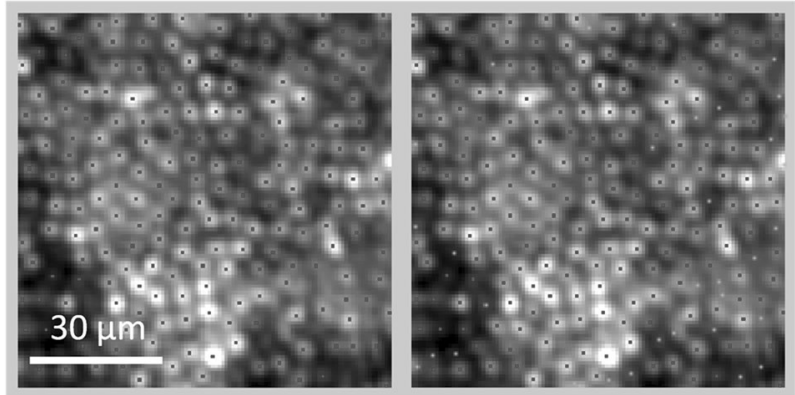

Fig. 2 Adaptative optics cone count. Right: cone counted automatically with the manufacturer's software (AO detect v0.2, Imagine Eyes, Orsay, France). Each red dot corresponds to a cone. Left: Manually corrected cone count. The cells added appear in blue

respectively). The annual cone loss estimated by the slope of the regression line was 104 cells $/ \mathrm{mm}^{2}$ per year with the automated counting and 116 cells $/ \mathrm{mm}^{2}$ with the manual counting (Fig. 3).

The comparison of cone density between the four age groups showed a significant difference between groups whether for automated or manual counting (ANOVA, $p<0.01$ ). (Fig. 4). The intersubject variability in cone density was noticeably high in overall age groups.

\section{Intrasubject variability}

The coefficient of variation between cone densities in fellow eyes reached $3.9 \%$ on average. There was no significant difference in the cone density measured in the nasal and temporal areas in the same subject (paired $t$ test, $p>0.05$ )

\section{Factors related to cone loss}

The multivariate analysis (Table 2 (supplemental)) showed that both $\mathrm{AL}$ and age were related to cone loss. However, neither choroid nor foveal thickness was a factor correlated with cone density changes.

\section{Discussion}

Aging in humans is associated with progressive and irreversible impairment of physiological functions, including vision. Older persons have deficits in both photopic and scotopic vision unrelated to a specific disease [25]. At the cellular level, the effect of age [26] was studied in normal retina and in age-related macular degeneration (AMD) [27]. 
Fig. 3 a Relationship between cone density obtained automatically and age. Cone density decreased with age $\left(R^{2}=0.16, p<0.01\right)$.

b Relationship between cone density obtained manually and age. Cone density decreased with age $\left(R^{2}=0.17, p<0.01\right)$
A

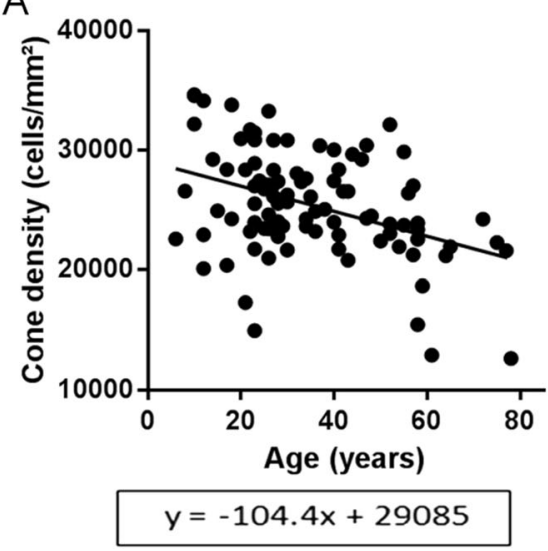

B

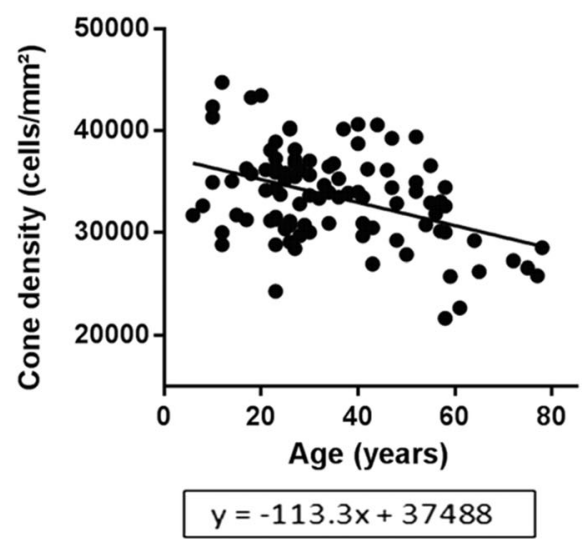

It appeared that the outer segments of photoreceptors become disorganized, and lipofuscin accumulates in cone inner segments with age $[28,29]$. It has been suggested that retinal pigment epithelium engorgement with lipofuscin impairs its ability to sustain photoreceptors, a phenomenon exacerbated in AMD [30]. Overall, pathways involved in stress response and energy metabolism seem to play key roles in retinal aging [31]. Significant differences in gene expression profiles between young human retinas and retinas in elderly subjects have been observed, with genes involved in cell growth and protein processing preferentially expressed in retinas of young individuals, whereas genes involved in stress response were expressed in elderly subjects [32].

Nevertheless, quantitative data describing senescent changes in human retinal photoreceptor density are limited. Histological studies have already suggested a loss of rods occurring early in adult life and a steady decrease in cone density throughout adulthood [33], in the far retina but not in the foveal peak. In a postmortem study, Curcio et al. [34] concluded that there were no changes in cone density throughout the age span, whereas rod density decreased by $30 \%$.

In contrast, in the current study, we found a moderate but significant decrease in perifoveal cones with age. These findings might not be as divergent as they seem and several hypotheses can explain the differences observed. First, histological studies examine eyes mostly from diseased or injured patients, hence technical problems to overcome, including tissue fixation and sample shrinkage [35], limiting the number of eyes studied. As a consequence, it is more difficult to obtain a homogeneous age group required for statistical comparison of cone density. For instance, a young patient who died from severe head trauma could have suffered commotio retinae [36], which might reduce the cone density measured postmortem, possibly removing the difference with the cone count of an older patient. In addition, the very principle of cone imaging with adaptive optics should be taken into consideration in interpreting these results.

In fact, the AO reflectivity of the cone photoreceptors is a result of the light-guiding properties of the cone, known as the Stiles-Crawford effect [37]. The backscatter light implies being redirected by the external segments of the cones. It is possible that AO preferentially images healthy cones, while overlooking cones that are impaired by age, the latter still being counted in flat-mounted retinae. The recent introduction of the nonconfocal split detector in $\mathrm{AO}$ devices [38], which images the inner segments of cones, will help differentiate healthy from impaired cones lacking external segments.

In a study of factors affecting human cone photoreceptor density measured by AOSLO, Park et al. [22] also observed a trend towards a slightly negative correlation between age and density $(r=-0.12)$, but the latter did not reach statistical significance $(p=0.14)$. They reported a cone density at 500 $\mu \mathrm{m}$ eccentricity from the fovea higher than that measured in the current study (32,000 vs. 26,166 cones/mm [24]).

Differences in reflectivity contrast and resolution, among other parameters, have already been reported between images obtained with AOSLO and flood illumination AO devices [39]. In addition, the counting protocol varies in different studies [40] (i.e., software, window size, counting algorithm [18, 41], etc.). It should be noted that we included a higher proportion of patients older than 50 years, which could also have contributed to a smaller average density.

Considering that there was a statistically significant difference in cone density between the youngest vs. the oldest and the under 35 year olds vs. the over 50 year olds, one could hypothesize that cone density loss is not regular over the lifespan but rather follows two phases with a plateau followed by decay. Interestingly, a study measuring the number of foveal photoreceptor nuclei found a reduction in donors older than 90 years of age compared to midlife donors 

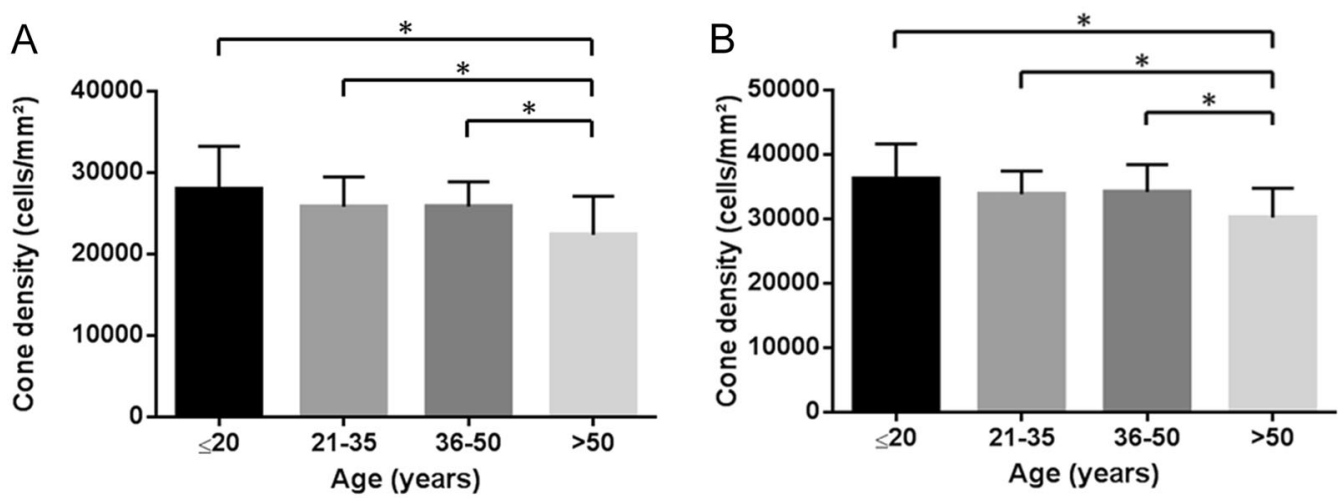

Fig. 4 a Comparison Automated cone counts (one-way ANOVA, ${ }^{*} p<0.05$ ). b Comparison between the age groups. Manual cone counts (one-way ANOVA, $* p<0.05$ )

[42]. It has been suggested that this reduction could be due to a selective loss of a specific population of cones, that is, bluesensitive cones. The choroid consists mostly of blood vessels, it supplies the outer retina, and choroidal defects cause degenerative changes and neovascularization. It also ensures thermoregulation, adjustment of the position of the retina by changes in choroidal thickness, and secretion of growth factors [43]. To our knowledge, this is the first study to compare choroidal thickness with the corresponding cone metrics. The absence of a relationship between choroidal thickness and foveal cone density may stem from having studied healthy subjects with the thinnest choroid measured at $109 \mu \mathrm{m}$, which could be sufficient to ensure its support role. However, studying cone density in diseased choroid with a swept-source OCT performing choroidal mapping [3] would be instructive to assess the extent to which choroidal thinning spares the underlying cones. In a qualitative note, we found no modification of the cone packing mosaic with age. However, even if all the participants had 20/20 vision, this does not exclude subclinical visual acuity impairment with age that could have been more sensitive tools such as spatial contrast sensitivity measurement [44].

This study has limitations, especially when it comes to imaging elderly patients. In this group many factors generate high-order aberrations that alter the quality of the retinal image. Factors such as poor quality of the tear film, cornea guttata, or mild cataract cannot be overcome, substantially reducing the number of imageable patients in this age subgroup. Furthermore, a longitudinal follow-up of the study would have reinforced the findings. Finally, the findings should not be extrapolated to other retinal areas or to retinal conditions where aging could have a much more significant impact.

AO showed a slight but significant decline in perifoveal cone density, independent of the choroidal thinning observed with age, with relative preservation of the cone mosaic. In a context of continuous extension of life expectancy in developed countries, the precise functional consequences of these findings should be investigated

\section{Summary}

\section{What was known before}

- In vivo data assessing the relationship between photoreceptor and aging are scarce.

- It is important to study aging process in healthy eyes in order to improve our knowledge of age-related diseases.

\section{What this study adds}

- This study provides a better understanding of the relationship between photoreceptor loss and ageing.

\section{Compliance with ethical standards}

Conflict of interest The authors declare that they have no conflict of interest.

\section{References}

1. Morrison JH, Hof PR. Life and death of neurons in the aging brain. Science (New York, NY). 1997;278:412-19.

2. Audo I, Sanharawi MEl, Vignal-Clermont C, Villa A, Morin A, Conrath J, Fompeydie D, et al. Foveal damage in habitual poppers users. Arch Ophthalmol. 2011;129:703-8. https://doi.org/10. 1001/archophthalmol.2011.6

3. Michalewski J, Michalewska Z, Nawrocka Z, Bednarski M, Nawrocki J. Correlation of choroidal thickness and volume measurements with axial length and age using swept-source optical coherence tomography and optical low-coherence reflectometry. Biomed Res Int. 2014;2014:639160.

4. Bowd C, Zangwill LM, Blumenthal EZ, Vasile C, Boehm AG, Gokhale PA, Mohammadi K, Amini P, Sankary TM, Weinreb. RN. Imaging of the optic disc and retinal nerve fiber layer: the effects of age, optic disc area, refractive error, and gender. J Opt Soc Am A. 2002;19:197-207. 
5. Eriksson U, Alm A. Macular thickness decreases with age in normal eyes: a study on the macular thickness map protocol in the stratus OCT. Br J Ophthalmol. 2009;93:1448-52. https://doi.org/ 10.1136/bjo.2007.131094

6. Manjunath V, Mohammad T, Fujimoto JG, Duker JS. Choroidal thickness in normal eyes measured using cirrus HD optical coherence tomography. Am J Ophthalmol. 2010;150:325-9.e1. https://doi.org/10.1016/j.ajo.2010.04.018

7. Chui TYP, Song H, Clark CA, Papay JA, Burns SA, Elsner AE. Cone photoreceptor packing density and the outer nuclear layer thickness in healthy subjects. Invest Ophthalmol Vis Sci. 2012;53:3545-53.

8. Obata R, Yanagi Y. Quantitative analysis of cone photoreceptor distribution and its relationship with axial length, age, and early age-related macular degeneration. PLoS ONE. 2014;9:e91873.

9. Lombardo M, Lombardo G, Schiano Lomoriello D, Ducoli P, Stirpe M, Serrao S. Interocular symmetry of parafoveal photoreceptor cone density distribution. Retina (Phila, PA). 2013;33:1640-9.

10. Muthiah MN, Gias C, Chen FK, Zhong J, McClelland Z, Sallo FB, et al. Cone photoreceptor definition on adaptive optics retinal imaging. Br J Ophthalmol. 2014;98:1073-9.

11. Li KY, Tiruveedhula JS, Roorda A. Intersubject variability of foveal cone photoreceptor density in relation to eye length. Invest Ophthalmol Vis Sci. 2010;51:6858-67. https://doi.org/10.1167/ iovs.10-5499

12. Choi SS, Zawadzki RJ, Lim MC, Brandt JD, Keltner JL, Doble N, Werner JS. Evidence of outer retinal changes in glaucoma patients as revealed by ultrahigh-resolution in vivo retinal imaging. $\mathrm{Br} \mathrm{J}$ Ophthalmol. 2011;95:131-41. https://doi.org/10.1136/bjo.2010. 183756

13. Duncan JL, Zhang Y, Gandhi J, Nakanishi C, Othman M, Branham KEH, Swaroop A, Roorda A. High-resolution imaging with adaptive optics in patients with inherited retinal degeneration. Invest Ophthalmol Vis Sci. 2007;48:3283-91. https://doi.org/10. 1167/iovs.06-1422

14. Zayit-Soudry S, Jacque LD, Syed R, Menghini M, Austin JR. Cone structure imaged with adaptive optics scanning laser ophthalmoscopy in eyes with nonneovascular age-related macular degeneration. Invest Ophthalmol Vis Sci. 2013;54:7498-509. https://doi.org/10.1167/iovs.13-12433

15. Chen Y, Ratnam K, Sundquist SM, Lujan B, Ayyagari R, Gudiseva VH, Roorda A, Jacque LD. Cone photoreceptor abnormalities correlate with vision loss in patients with Stargardt disease. Invest Ophthalmol Vis Sci. 2011;52:3281-92. https://doi. org/10.1167/iovs.10-6538

16. Gocho K, Sarda V, Falah S, Sahel J-Al, Sennlaub F, Benchaboune M, Ullern M, Paques M. Adaptive optics imaging of geographic atrophy. Invest Ophthalmol Vis Sci. 2013;54:3673-80. https://doi.org/10.1167/iovs.12-10672

17. Ooto S, Hangai M, Sakamoto A, Tsujikawa A, Yamashiro K, Ojima Y, Yamada Y, et al. High-resolution imaging of resolved central serous chorioretinopathy using adaptive optics scanning laser ophthalmoscopy. Ophthalmology. 2010;117:1800-9.e1-2. https://doi.org/10.1016/j.ophtha.2010.01.042

18. Ooto S, Hangai M, Takayama K, Ueda-Arakawa N, Hanebuchi M, Yoshimura N. Photoreceptor damage and foveal sensitivity in surgically closed macular holes: an adaptive optics scanning laser ophthalmoscopy study. Am J Ophthalmol. 2012;154:174-86.e2. https://doi.org/10.1016/j.ajo.2012.01.031

19. Saleh, $M$, Debellemanière $G$, Meillat $M$, Tumahai $P$, Bidaut Garnier M, Flores M, Schwartz C, Delbosc B. Quantification of cone loss after surgery for retinal detachment involving the macula using adaptive optics. Br J Ophthalmology. 2014;98 (10):1343-8. https://doi.org/10.1136/bjophthalmol-2013-304813
20. Bae EJ, Kim KR, Tsang SH, Park SP, Chang S. Retinal damage in chloroquine maculopathy, revealed by high resolution imaging: a case report utilizing adaptive optics scanning laser ophthalmoscopy. Korean J Ophthalmol. 2014;28:100-7. https://doi.org/10. 3341/kjo.2014.28.1.100

21. Song H, Chui TYP, Zhong Z, Elsner AE, Stephen AB. Variation of cone photoreceptor packing density with retinal eccentricity and age. Invest Ophthalmol Vis Sci. 2011;52:7376-84. https://doi. org/10.1167/iovs.11-7199

22. Park S, Pyo JKC, Vivienne G, Stephen HT, Stanley C. A study of factors affecting the human cone photoreceptor density measured by adaptive optics scanning laser ophthalmoscope. Exp Eye Res. 2013;108:1-9. https://doi.org/10.1016/j.exer.2012.12.011

23. Bidaut Garnier M, Flores M, Debellemanière G, Puyraveau M, Tumahai P, Mathieu M, Schwartz C, Montard M, Delbosc B, Saleh M. Reliability of cone counts using an adaptive optics retinal camera. Clin Exp Ophthalmol. 2014;42(9):833-40. https://doi. org/10.1111/ceo.12356

24. Gartner S, Henkind P. Aging and degeneration of the human macula. 1. Outer Nuclear layer and photoreceptors. Br J Ophthalmol. 1981;65:23-28.

25. NATO Advanced Research Workshop on the Changing Visual System: From Early to Late Stages of Life-Maturation and Aging in the Central Nervous System, and North Atlantic Treaty Organization. In: Bagnoli P, Hodos W, editors. The changing visual system: maturation and aging in the central nervous system. NATO ASI Series, v. 222. New York: Plenum Press; 1991. p. 119-36.

26. Nag TC, Wadhwa S. Ultrastructure of the human retina in aging and various pathological states. Micron. 2012;43:759-81.

27. Adler R, Curcio C, Hicks D, Price D, Wong F. Cell death in agerelated macular degeneration. Mol Vis. 1999;5:31.

28. Tucker GS. Refractile bodies in the inner segments of cones in the aging human retina. Invest Ophthalmol Vis Sci. 1986;27:708-15.

29. Iwasaki M, Inomata H. Lipofuscin granules in human photoreceptor cells. Invest Ophthalmol Vis Sci. 1988; 29:671-79.

30. Curcio CA. Photoreceptor topography in ageing and age-related maculopathy. Eye. 2001;15:376-83. https://doi.org/10.1038/eye. 2001.140

31. Eckmiller MS, Marion S. Defective cone photoreceptor cytoskeleton, alignment, feedback, and energetics can lead to energy depletion in macular degeneration. Prog Retin Eye Res. 2004;23:495-522. https://doi.org/10.1016/j.preteyeres.2004.04.005

32. Yoshida S, Beverly MY, Hiriyanna S, Swaroop A. Microarray analysis of gene expression in the aging human retina. Invest Ophthalmol Vis Sci. 2002;43:2554-60.

33. Panda-Jonas S, Jonas JB, Jakobczyk-Zmija M. Retinal photoreceptor density decreases with age. Ophthalmology. 1995; 102:1853-59.

34. Curcio CA, Millican CL, Allen KA, Kalina RE. Aging of the human photoreceptor mosaic: evidence for selective vulnerability of rods in central retina. Invest Ophthalmol Vis Sci. 1993;34:3278-96

35. Marshall J. Ageing changes in human cones. In: ShimuzuK, editor. Ophthalmology. Vol 1. Proc. XXIII International Congr (Kyoto); 1979. p. 375-8

36. Noia LdaC, Berezovsky A, Freitas Dd, Sacai PY, Salomão SR. Clinical and electroretinographic profile of commotio retinae. Arq Bras Oftalmol. 2006;69:895-906.

37. Westheimer G. Directional sensitivity of the retina: 75 years of Stiles-Crawford effect. Proc Biol Sci. 2008;275:2777-86.

38. Scoles D, Sulai YN, Langlo CS, Fishman GA, Curcio CA, Carroll $\mathrm{J}$, et al. In vivo imaging of human cone photoreceptor inner segments. Invest Ophthalmol Vis Sci. 2014;55:4244-51. 
39. Lombardo M, Serrao S, Ducoli P, Lombardo G. Eccentricity dependent changes of density, spacing and packing arrangement of parafoveal cones. Ophthalmic Physiol Opt. 2013;33: 516-26.

40. Garrioch R, Langlo C, Dubis AM, Cooper RF, Dubra A, Carroll J. Repeatability of in vivo parafoveal cone density and spacing measurements. Optom Vis Sci. 2012;89:632-43.

41. Lombardo M, Serrao S, Ducoli P, Lombardo G. Influence of sampling window size and orientation on parafoveal cone packing density. Biomed Opt Express. 2013;4: $1318-31$.

42. Feeney-Burns L, Burns RP, Gao CL. Age-related macular changes in humans over 90 years old. Am J Ophthalmol. 1990;109:265-78.

43. Nickla DL, Wallman J. The multifunctional choroid. Prog Retin Eye Res. 2010;29:144-68.

44. Owsley C. Contrast sensitivity. Ophthalmol Clin N Am. 2003; 16:171-7. 